\title{
A self-renewing, tissue-engineered vascular graft for arterial reconstruction
}

\author{
Kei Torikai, MD, ${ }^{a}$ Hajime Ichikawa, MD, PhD, ${ }^{a}$ Koichiro Hirakawa, MS, ${ }^{b}$ Goro Matsumiya, MD, PhD, ${ }^{a}$ Toru Kuratani, MD, \\ $\mathrm{PhD},{ }^{a}$ Shigemitsu Iwai, MD, PhD, ${ }^{a}$ Atsuhiro Saito, PhD, ${ }^{a}$ Naomasa Kawaguchi, PhD, ${ }^{c}$ Nariaki Matsuura, MD, PhD, ${ }^{c}$ and \\ Yoshiki Sawa, MD, PhD ${ }^{a}$
}

Supplemental material is available online.
From the Division of Cardiovascular Surgery, Department of Surgery, Osaka University Graduate School of Medicine, Osaka, Japan ${ }^{\mathrm{a}}$; Senko Medical Instrument Manufacturing Co Ltd, Tokyo, Japan ${ }^{\text {b }}$; and the Department of Pathology, Osaka University School of Allied Health Science, Osaka, Japan. $^{c}$

We developed a tissue-engineered vascular graft for arterial reconstruction to facilitate renewing of autologous tissue without pretreatment and evaluated its utility in a porcine model. The graft showed morphologic evidence of good in situ cellularization, satisfactory durability against arterial pressure for 12 months, and potential to acquire vasomotor responsiveness.

Supported by a grant from the Ministry of Economy, Trade, and Industry in the Japanese government.

Received for publication Dec 29, 2006; revisions received May 15, 2007; accepted for publication June 25, 2007.

Address for reprints: Yoshiki Sawa, MD, PhD, 2-2 Yamadaoka, Suita, Osaka, 5650871, Japan (E-mail: sawa@surg1.med. osaka-u.ac.jp).

J Thorac Cardiovasc Surg 2008;136:37-45 0022-5223/ $\$ 34.00$

Copyright $\odot 2008$ by The American Association for Thoracic Surgery

doi:10.1016/j.jtcvs.2007.06.039
Objective: Various tissue-engineered vascular grafts have been studied to overcome the clinical disadvantages of conventional prostheses. Previous tissue-engineered vascular grafts have generally required preoperative cellular manipulation or use of bioreactors to improve performance, and their mechanical properties have been insufficient. We focused on the concept of in situ cellularization and developed a tissue-engineered vascular graft for arterial reconstruction that would facilitate renewal of autologous tissue without any pretreatment.

Methods: The graft comprised an interior of knitted polyglycolic acid compounded with collagen to supply a scaffold for tissue growth and an exterior of woven polyL-lactic acid for reinforcement. All components were biocompatible and biodegradable, with excellent cellular affinity. The grafts, measuring $10 \mathrm{~mm}$ in internal diameter and $30 \mathrm{~mm}$ in length, were implanted into porcine aortas, and their utility was evaluated to 12 months after grafting.

Results: All explants were patent throughout the observation period, with no sign of thrombus formation or aneurysmal change. Presence in the neomedia of endothelialization with proper integrity and parallel accumulation of functioning smooth muscle cells, which responded to vasoreactive agents, was confirmed in an early phase after implantation. Sufficient collagen synthesis and lack of elastin were quantitatively demonstrated. Dynamic assessment and long-term results of the in vivo study indicated adequate durability of the implants.

Conclusion: The graft showed morphologic evidence of good in situ cellularization, satisfactory durability to withstand arterial pressure for 12 postoperative months, and the potential to acquire physiologic vasomotor responsiveness. These results suggest that our tissue-engineered vascular graft shows promise as an arterial conduit prosthesis.

A rtificial vascular grafts manufactured from synthetic materials, for example polyester and expanded polytetrafluoroethylene (ePTFE), have been routinely used to reconstruct blood flow in patients with various cardiovascular disorders. Conventional grafts have clinically shown satisfactory durability; however, they still have several disadvantages, such as thrombogenicity, late stenosis and occlusion from intimal hyperplasia (especially in small caliber grafts), susceptibility to infection, and lack of growth potential. ${ }^{1,2}$ To overcome these limitations in the search for an ideal artificial graft, various tissue-engineered vascular grafts (TEVGs) have been developed. ${ }^{3}$ The utility and the clinical experience of these new grafts have been reported. ${ }^{4-6}$ In TEVGs, a biodegradable polymer or a decellularized biomaterial is commonly used as a scaffold to enable host cells to rebuild the vessel architecture, and autologous cell seeding and culture or growth with bioreactors before the operation is usually necessary to improve their antithrombogenicity and performance. ${ }^{1,2}$, 7-11 These pretreatments involve complicated and invasive procedures, potentially leading to infection, and also require a certain period to complete, which means that pretreated TEVGs are not always available or may be unsuitable for an emergency 


\section{Abbreviations and Acronyms}

ePTFE = expanded polytetrafluoroethylene

HUVEC = human umbilical vein endothelial cell

PGA $=$ polyglycolic acid

PLLA = poly-L-lactic acid

SMC $=$ smooth muscle cell

SNP = sodium nitroprusside

TEVG $=$ tissue-engineered vascular graft case. ${ }^{5}$ Furthermore, use of TEVGs has been mostly limited to low-pressure conditions because of their poor durability. $5,6,12$

For these reasons, we focused on the concept of in situ cellularization, whereby a population of living host cells is achieved in situ without any pretreatment by supplying a scaffold to support tissue self-renewal. This scaffold acts as an in situ incubator and bioreactor. Chen and colleagues ${ }^{13}$ realized this concept in the regeneration of bovine chondrocytes by using a biocompatible material with excellent cellular attachment properties. We recently developed two promising novel patches for cardiovascular repair, a poly(lactic-co-glycolic acid) and collagen patch ${ }^{14}$ and a tissue-engineered patch, ${ }^{15}$ both of which showed good in situ cellularization in vessel wall reconstruction of large animal models. We hypothesized that an entire tubular graft fabricated from our patch would be an ideal vascular prosthesis that could acquire favorable morphologic features and also intrinsic vascular function matched to the native artery by means of in situ cellularization. This kind of TEVG has not yet been reported.

In this study, we designed a TEVG for arterial reconstruction that was expected to facilitate regeneration of autologous tissue without any pretreatment and assessed in situ cellularization after implantation in a porcine model. We also evaluated whether the implanted TEVG possessed adequate durability to withstand the hemodynamic stress of the systemic circulation in a long-term follow-up model and had acquired the physiologic vasoreactivity inherent in an artery.

\section{Materials and Methods}

\section{Graft Design and Cellular Affinity}

The new vascular prosthesis was made from a tissue-engineered patch $^{15}$ recently developed in our laboratory with greater biocompatibility and mechanical strength than previous materials used for cardiovascular repair. The patch has a three-layered structure: the interior (luminal side) is composed of knitted polyglycolic acid (PGA) compounded with collagen microsponge, the middle layer is polycaprolactone, and the exterior is composed of woven poly-L-lactic acid (PLLA; Figure 1A). It was provided by Senko Medical Instrument Mfg Co Ltd (Tokyo, Japan). All these materials are biocompatible and biodegradable and have received Food and Drug Administration clearance. The internal 3-dimensional porous structure of knitted PGA and collagen microsponge is expected to play a crucial role as a scaffold promoting in situ cellularization. The tightly woven layer of PLLA on the outside reinforces the patch,
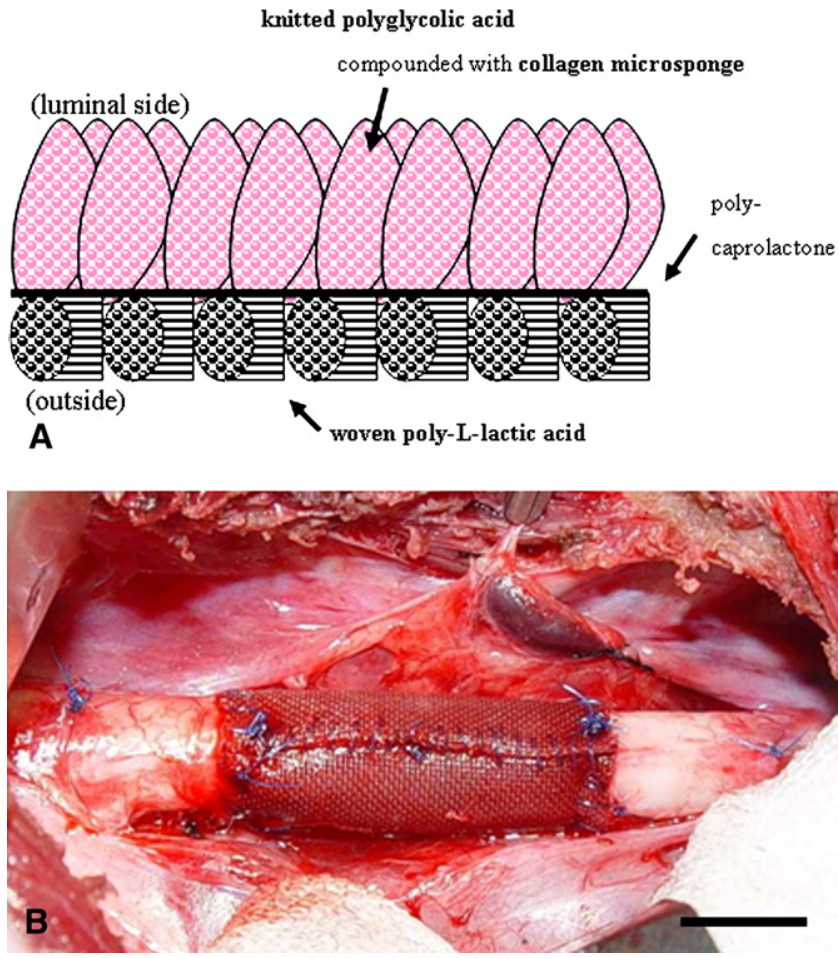

Figure 1. A, Scaffold design of polyglycolic acid/poly---lactic acid graft. Graft wall had three-layered structure: luminal side, knitted polyglycolic acid compounded with collagen microsponge; middle layer, polycaprolactone; outside, woven poly-L-lactic acid. B, Implantation of graft into porcine aorta. Scale bar represents $10 \mathrm{~mm}$.

and a polycaprolactone film binds the PGA and PLLA layers to each other. Degradation tests $\left(37^{\circ} \mathrm{C}\right)$ of the synthetic polymers, mainly involving hydrolytic reactions, demonstrated that the mechanical strength of PGA was no longer adequate by 3 weeks, whereas PLLA was degraded so slowly that its strength was maintained through 6 months.

Cell culture tests were performed to evaluate cellular affinity and proliferation in the materials used in this study. Two lines of cells, human umbilical vein endothelial cells (HUVEC; Cambrex Corporation, East Rutherford, NJ) and murine fibroblasts (NIH3T3; American Type Culture Collection, Manassas, Va) were seeded at a density of 300 cells $/ \mathrm{mm}^{2}$ onto knitted PGA with or without collagen microsponge, woven PLLA, and ePTFE (GoreTex; Japan GoreTex Inc, Tokyo, Japan) $(\mathrm{n}=6)$, then cultured at $37^{\circ} \mathrm{C}$ at $100 \%$ humidity and in a $5 \%$ carbon dioxide atmosphere. The culture medium was Dulbecco modified Eagle medium (Gibco; Invitrogen Corporation, Carlsbad, Calif) supplemented with $10 \%$ fetal bovine serum (Sigma Chemical Co, St Louis, Mo) and $1 \%$ penicillin and streptomycin (Invitrogen). After 3 days of culture, the number of cells attached to each material was counted by a water-soluble tetrazolium assay (Dojindo Laboratories, Kumamoto, Japan).

\section{Implantation in Porcine Models}

A tissue-engineered patch was manually formed into a tubular shape with continuous 5-0 Prolene sutures (Ethicon, Inc, Somerville,NJ). 
This graft (PGA/PLLA graft) measured $10 \mathrm{~mm}$ in internal diameter and $30 \mathrm{~mm}$ in length. We implanted these grafts into the thoracic descending aorta of Crown minipigs (12-20 kg; Figure 1B). General anesthesia was induced by means of intramuscular bolus injection of 20-mg/kg ketamine (Sankyo Co, Tokyo, Japan) and 2-mg/kg xylazine (Bayer Medical Co, Tokyo, Japan) and maintained with a continuous intravenous infusion of $8-\mathrm{mg} /(\mathrm{kg} \cdot \mathrm{h})$ propofol (AstraZeneca, Osaka, Japan) and additional injections of ketamine at 10 $\mathrm{mg} /(\mathrm{kg} \cdot \mathrm{h})$. The aorta was approached through a left posterolateral thoracotomy. After systemic heparinization (100 IU/kg), the aorta was clamped and resected, and simultaneously a shunt circuit was established to prevent paraplegic complications. Proximal and distal anastomoses were then performed with 5-0 Prolene running sutures. No anticoagulants or antiplatelet agents were given after the operation. The animals were randomly divided into five groups, and the vascular prostheses were retrieved when the animals were killed at $1(\mathrm{n}=2), 2(\mathrm{n}=4), 4(\mathrm{n}=5), 6$, and 12 months $(\mathrm{n}=4)$ after implantation. Five ePTFE grafts of the same size as the PGA/PLLA grafts were implanted in a similar manner and harvested $(2,4$, and 6 months after implantation, $\mathrm{n}=1, \mathrm{n}=3$, and $\mathrm{n}=1$, respectively) as controls. All pigs received humane care and treatment in accordance with the "Guide for the Care and Use of Laboratory Animals" (www.nap.edu/catalog/5140.html).

\section{Tissue Assessment}

Explanted grafts were opened longitudinally and then photographed as gross specimens. The macroscopic endothelial coverage rate on the inner surfaces of the grafts was calculated with the image analysis software package Scion Image (Scion Corporation, Frederick, Md). Part of the specimen prepared in a typical fashion was analyzed by scanning electron microscopy (S-800; Hitachi, Ltd, Tokyo, Japan). We also measured the wall thickness of the neotissue, which was defined as regenerated tissue comprising newly organized autologous cells inside the PLLA layer. A longitudinal section of the harvested grafts was processed for histologic evaluation and was examined by double staining with hematoxylin and eosin and Victoria blue stains for elastic fibers. Immunohistologic staining was performed to identify cell types and matrices in the regenerated tissue. The antibodies used were monoclonal antibodies against $\alpha$-smooth muscle actin (DAKO Corporation, Carpenteria, Calif) and collagen type IV (DAKO) and a polyclonal antibody against von Willebrand factor (rabbit; DAKO) for endothelial cells.

The content of representative extracellular matrices was biochemically quantified with the regenerated tissue alone. The collagen content was measured with a 4-hydroxyproline assay. The elastin content was measured by determining the dry weight of the insoluble material after delipidation in acetone-diethylether and solubilization in $0.1-\mathrm{N}$ sodium hydroxide at $98^{\circ} \mathrm{C}$. We compared the measured amounts in each group with those from native aortas $(\mathrm{n}=19)$.

The maximal tensile strength of residual PLLA layers taken from the explants was measured with a TENSILON mechanical tester (ORIENTEC Co, Tokyo, Japan). Samples of size $20 \times 10 \mathrm{~mm}$ were used, with an initial crosshead span of $10 \mathrm{~mm}$ and a speed of $10 \mathrm{~mm} / \mathrm{min}$. The strength was represented in newtons.

The vasomotor responsiveness of the retrieved PGA/PLLA grafts was examined both qualitatively and quantitatively. Prostheses explanted 2 months after implantation $(n=2)$ were immediately placed for preservation into ice cold Krebs solution. ${ }^{16}$ The regenerated tissue was carefully dissected from the explants and cut into cross-sectional segments of $20 \times 5 \mathrm{~mm}$. We observed the morphologic changes in suspended segments at $37^{\circ} \mathrm{C}$ on direct administration of $10^{-3}-\mathrm{mol} / \mathrm{L}$ norepinephrine (Sankyo) and sodium nitroprusside (SNP; Maruishi Pharmaceutical Co, Osaka, Japan) as previously described. ${ }^{17}$ To quantify the responses, standard isometric tension experiments were performed. ${ }^{16}$ Briefly, $10 \times 5$ $\mathrm{mm}$ segments prepared from 2- and 12-month models were stretched to an initial tension of $8 \mathrm{~g}$ and then allowed to equilibrate for 2 hours in an organ chamber $\left(37^{\circ} \mathrm{C}\right)$ containing oxygenated Krebs solution. All the following experiments were done in the presence of $10^{-5}-\mathrm{mol} / \mathrm{L}$ indomethacin (Sigma). Contractile responses to increasing concentrations of norepinephrine were measured and expressed in milligrams. To investigate endothelium-independent relaxation, the segments were contracted in advance with $10^{-5} \mathrm{~mol} / \mathrm{L}$ norepinephrine so that a stable baseline of contraction was achieved, then the dose-response curve to SNP was determined. The relaxation responses were expressed as the percentage reduction in the tension produced by $10^{-5}$-mol/L norepinephrine. Matched segments of native aortas $(n=5)$ were used as controls.

\section{Statistical Analysis}

All results are expressed as mean \pm SEM. Cell culture tests were analyzed with the Student $t$ test. Comparisons of measured data among multiple groups were performed with appropriate analysis of variance and the Tukey-Kramer post hoc test. In all statistical analyses, the commercially available software package StatView (SAS Institute, Inc, Cary, NC) was used.

\section{Results}

\section{Cellular Affinity}

With both cell lines, culture tests showed that the number of cells attached to knitted PGA with collagen microsponge was significantly greater than that without collagen (HUVEC with collagen $1566 \pm 191$ cells $/ \mathrm{mm}^{2}$ and without collagen $1168 \pm 137$ cells $/ \mathrm{mm}^{2}, P=.0020$, NIH3T3 with collagen $2337 \pm 550$ cells $/ \mathrm{mm}^{2}$ and without collagen $1859 \pm 342$ cells $\left./ \mathrm{mm}^{2}, P=.0418\right)$. This suggests that the presence of compounded collagen microsponge increased the cellular adhesion properties of knitted PGA. The cellular affinity of woven PLLA (HUVEC $716 \pm 56$ cells $/ \mathrm{mm}^{2}$, NIH3T3 $1517 \pm 270$ cells $/ \mathrm{mm}^{2}$ ) was less than that of PGA. The numbers of cells attached to ePTFE were $231 \pm 73$ and $591 \pm 151$ cells/mm ${ }^{2}$ for HUVEC and NIH3T3, respectively, indicating that PGA and PLLA have a significantly higher cellular affinity than ePTFE. These results were verified by scanning electron microscopy (data not shown).

\section{Implantation Results}

All the operations were completed successfully, with negligible bleeding through the walls of the implanted PGA/PLLA grafts after declamping of the aorta. All animals survived without any postoperative complications during the predetermined observation period. 


\section{Macroscopic Findings}

On gross inspection, each explanted graft was patent, with no sign of thrombus or aneurysm formation. The luminal surfaces of all grafts, even those in the 1-month group, were uniformly smooth and glistening, as in native aorta. The surfaces were completely endothelialized macroscopically. In ePTFE grafts, however, there was always some endothelial defect into which blood had soaked (Figure 2). The incidence of such defects was not associated with the duration of implantation, and the overall mean value of the endothelial coverage rate was $82.6 \% \pm 21.0 \%(46.5 \%-97.0 \%)$. The implanted grafts were surrounded by fibrous connective tissue, and none of the grafts had a severely stiffened wall. The PGA component could not be found macroscopically, whereas the PLLA layers were obvious in all specimens.

\section{Morphologic Examinations}

Scanning electron micrography. Scanning electron micrography revealed that the luminal surfaces of the PGA/ PLLA grafts 2 months after implantation were covered with confluent and cobblestonelike cells that were oriented parallel to the direction of blood flow, although they were aligned irregularly in places (Figure 3, A). After 6 months, confluent endothelial cells with prominent nuclei formed an orderly alignment on the intima (Figure $3, B$ and $C$ ). In the macroscopic endothelial defects in ePTFE grafts, ePTFE fibrils were directly exposed to the lumen without any endothelial coverage; instead, a number of blood cells were attached to the defective surface (Figure 3,D).
Wall thickness of neotissue. The neotissue in the 2-month group had a thickness of approximately $1.5 \mathrm{~mm}$ at each point, although the measured values were somewhat widely distributed (Figure E1). After this time, there were no significant differences in thickness at any point among the groups, indicating no tendency for wall thickness to increase in a timedependent manner. When the three measured points were compared within each group, there were no significant differences.

Histologic and immunohistochemical examinations. The histologic findings in each model are shown in Figure 4. We mainly observed sections featuring the middle portion, into which transanastomotic ingrowth of autologous cells would be relatively difficult. Immunohistochemically, von Willebrand factor-positive endothelial monolayers were already confirmed along the whole length of the luminal surfaces at 1 month. Beneath the endothelium, numerous smooth muscle cells (SMCs) were piled up in parallel, as seen in native media. Such good cellularization with the two types of cells in PGA/PLLA grafts was similarly found in the other groups. Extracellular matrix in the subendothelial layers was stained faintly by Victoria blue, but the intensity of the stain increased with time, and fiberlike structures consistent with elastic fibers were detected in long-term models. The PGA component of the grafts was degraded and absorbed almost completely by 2 postoperative months, whereas the PLLA was relatively intact and maintained its layered structure to 12 months. In the early phase, mild infiltration of inflammatory cells and phagocytic phenomena
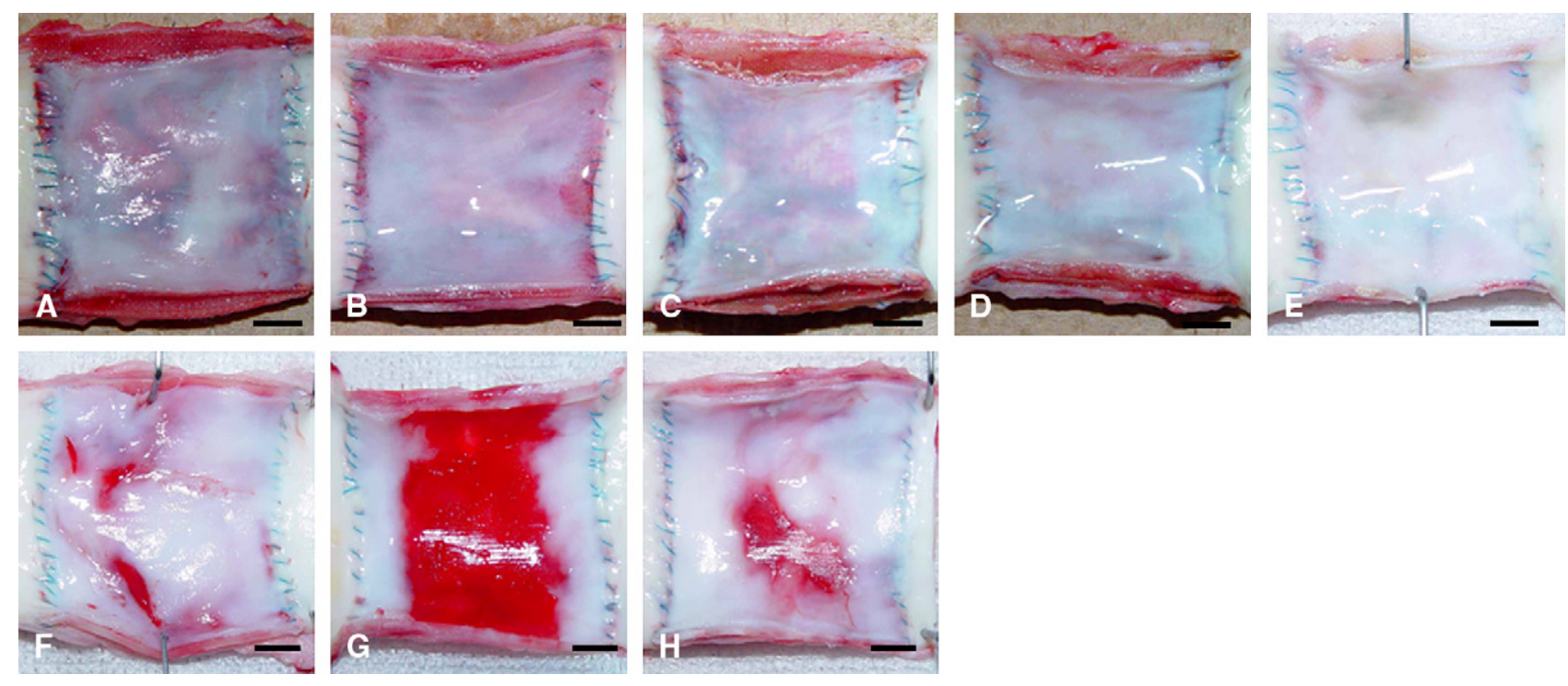

Figure 2. Upper row, Macroscopic findings of polyglycolic acid/poly-L-lactic acid grafts at 1 (A), 2 (B), 4 (C), 6 (D), and 12 (E) months after implantation. All explants were patent, with no sign of thrombus or aneurysm formation, and all surfaces were completely endothelialized. Lower row: macroscopic findings of expanded polytetrafluoroethylene grafts at 2 (F), 4 (G), and 6 (H) postoperative months. Endothelial defects were always detected. Scale bar represents $5 \mathrm{~mm}$. 

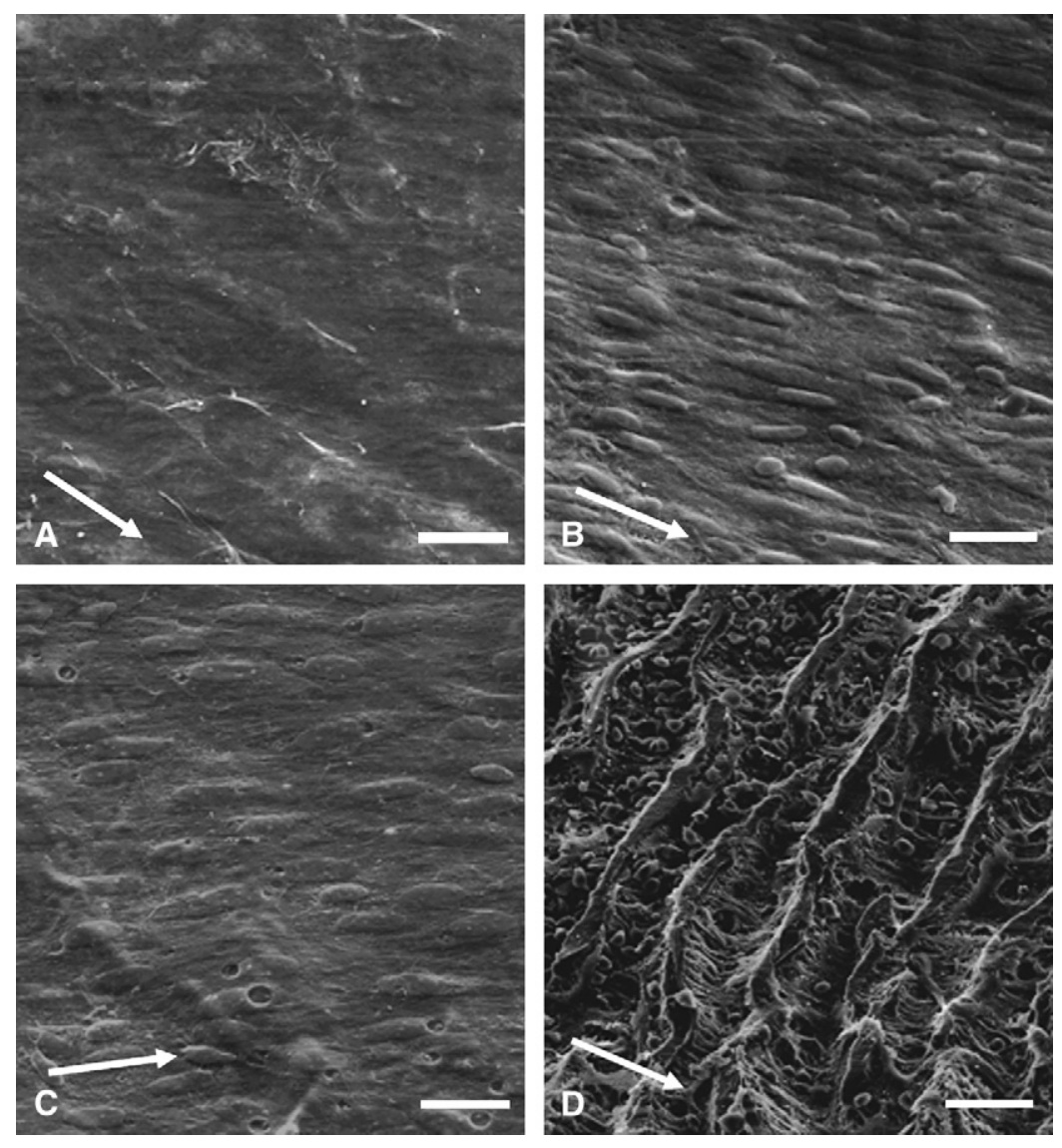

Figure 3. Scanning electron micrographs of luminal surfaces of polyglycolic acid/poly-L-lactic acid grafts 2 (A), 6 (B), and 12 (C) months after grafting. Surfaces were covered with confluent endothelial cell lining parallel to direction of blood flow. D, Scanning electron micrograph of endothelial defect in expanded polytetrafluoroethylene graft. Direct exposure of its fibrils to graft lumen was observed. Original magnification $\times 800$; scale bar, $20 \mu \mathrm{m}$. Arrows indicate direction of blood flow. were observed around the remnants of each material. Immunohistochemical testing for collagen type IV demonstrated positively stained layers, showing the existence of basal membrane, just below the endothelium in all the newly developed grafts. We also detected scattered positive staining in the neomedia, suggestive of vasa vasorum in the newly formed tissue (Figure E2). Microscopic examination of the ePTFE grafts showed only partial regeneration of autologous tissue on the surfaces.

\section{Biochemical Evaluation}

The 4-hydroxyproline assay revealed that the amount of collagen in the neotissue increased in proportion to the duration of implantation. At 6 months after implantation, the collagen content was equivalent to that in native aorta (Table 1). The elastin content increased slightly with time but after 4 months had reached a plateau at approximately a third of the native level (Table 1).

\section{Mechanical Properties}

The maximum tensile strengths of the remaining PLLA layers were $51.5 \pm 7.9,66.6 \pm 3.6,55.4 \pm 8.1,39.8 \pm$ 7.3, $26.6 \pm 19.4$, and $25.4 \pm 8.7 \mathrm{~N}$ before implantation $(\mathrm{n}=6)$ and in the 1-, 2-, 4-, 6-, and 12-month groups, respectively. The strength was significantly diminished in a timedependent manner. The mechanical strengths of the PLLA layers taken from 6- and 12-month specimens were greater than that of native aorta $(8.3 \pm 4.6 \mathrm{~N}, \mathrm{n}=6)$, although the differences were not statistically significant.

\section{Vascular Function}

As shown in Figure 5, A, samples derived from the 2-month group were suspended to form an arch shape. This shape became an open arch when norepinephrine was administered then closed on administration of SNP, indicating contraction and relaxation, respectively. These pharmacologic responses were repeatedly observed. The contractile activities of 2- and 12-month specimens in the standard isometric experiments are shown in Figure 5, B. Both groups exhibited doseresponse curves to norepinephrine, and the contractile force in the 12-month group was significantly greater than that in the 2-month group. The maximal contractile force (norepinephrine $\left.10^{-4} \mathrm{~mol} / \mathrm{L}\right)$ in the 12 -month model was $430 \pm$ $21 \mathrm{mg}, 16.3 \%$ of that in native aorta $(2642 \pm 189 \mathrm{mg})$ under the same conditions. We also observed dose-dependent 


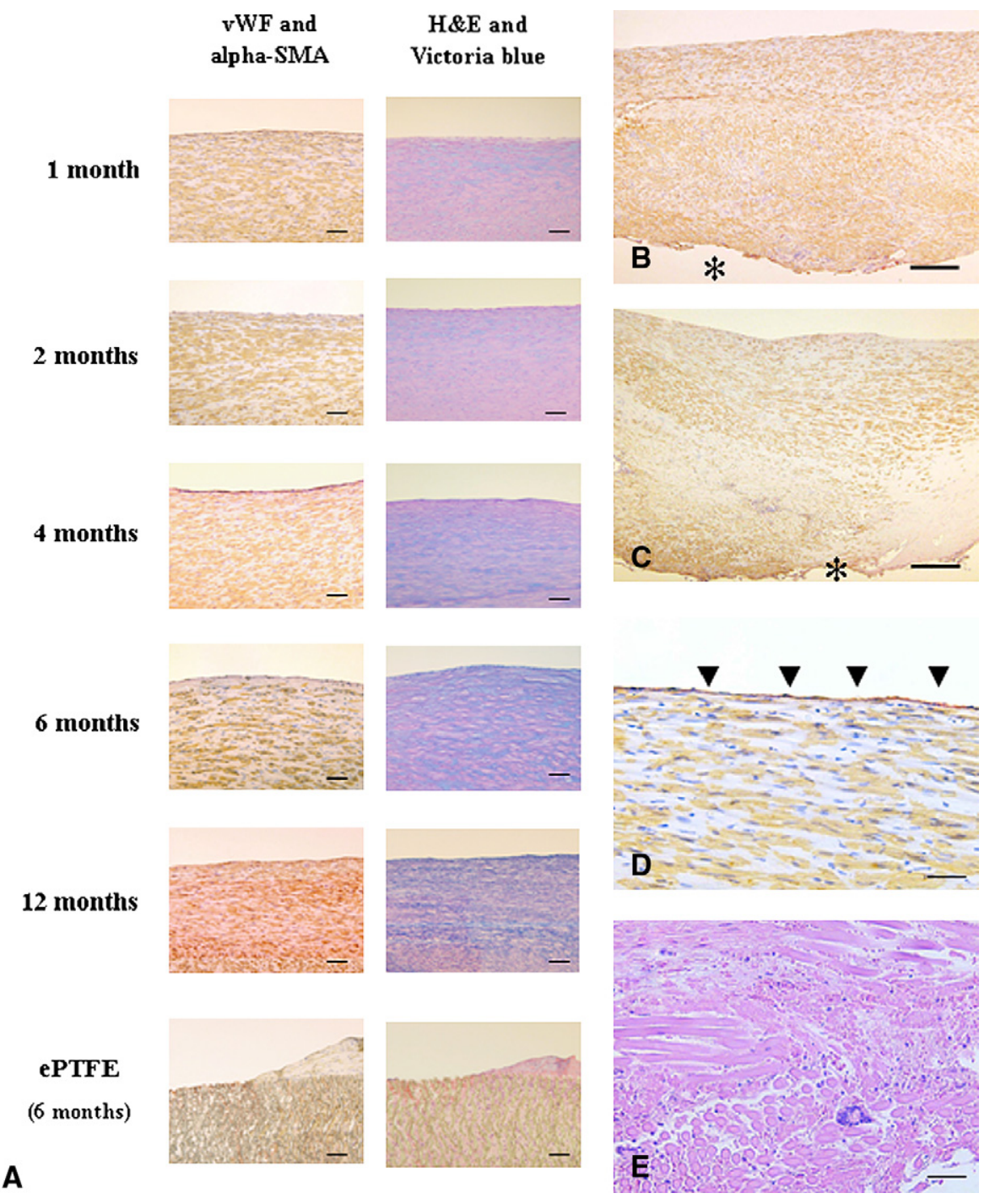

Figure 4. A, Histologic findings in each model. Left: immunohistochemical double staining for von Willebrand factor (vWF) and $\alpha$-smooth muscle actin (alpha-SMA). Right, Double staining with hematoxylin and eosin (H\&E) and Victoria blue stain. Immunohistochemical staining showed endothelial monolayers and parallel alignment of smooth muscle cells beneath endothelium in all polyglycolic acid/poly-L-lactic acid graft models. Victoria blue-positive fiberlike structures consistent with elastic fibers were detected in long-term models. With expanded polytetrafluoroethylene (ePTFE) grafts, good tissue regeneration was not observed. Original magnification $\times 100$; scale bar, $100 \mu \mathrm{m}$. B (2-month model) and C (12-month model) show all layers of implanted polyglycolic acid/poly-L-lactic acid grafts stained immunohistochemically. Asterisks represent space occupied by remaining poly-L-lactic acid layers. Original magnification $\times 40$; scale bar, $250 \mu \mathrm{m}$. D, Immunohistochemical findings in 2-month model. Arrowheads indicate endothelial monolayer. Original magnification $\times 200$; scale bar, $100 \mu \mathrm{m}$. $E$, Hematoxylin and eosin stain of polyglycolic acid/poly-L-lactic acid graft 1 month after implantation. Mild infiltration of inflammatory cells and phagocytic giant cell were observed around scaffold remnants in subendothelial layer. Original magnification $\times 200$; scale bar, $100 \mu \mathrm{m}$. relaxation of each sample in response to SNP (Figure 5, C). The percentage relaxations with $10^{-4} \mathrm{~mol} / \mathrm{L}$ SNP were $89.0 \% \pm 20.7 \%, 70.5 \% \pm 25.7 \%$, and $95.6 \% \pm 10.8 \%$ in the 2- and 12-month groups and the positive controls, respectively, with no significant differences. There was no significance difference in relaxation response to SNP between the 2- and 12-month groups.

The time course of the regeneration process in PGA/ PLLA grafts is shown in Table E1.

\section{Discussion}

Many strategies have recently been attempted to facilitate regeneration of autologous tissue on tissue-engineered materials. A major method is preoperative cellular manipulation. $^{5,9,10}$ Kaushal and coworkers ${ }^{9}$ demonstrated functional small diameter neovessels produced with endothelial progenitor cells. Another effective method is the use of a bioreactor. ${ }^{7-9,11}$ Campbell and associates ${ }^{8}$ sug- gested that the host's own peritoneal cavity is an effective bioreactor and showed that it is feasible to grow vascular prostheses within it. Also, Hoerstrup and colleagues ${ }^{11}$ demonstrated the growth capacity of cell-seeded TEVGs grown in vitro for 21 days under biomimetic conditions. Although we fully recognize the utility of these pretreatments, their

TABLE 1. Biochemical quantification of representative extracellular matrices

\begin{tabular}{lcc}
\hline \multicolumn{1}{c}{ Group } & Collagen content $(\mu \mathbf{g} / \mathbf{m g})$ & Elastin content $(\mu \mathbf{g} / \mathbf{m g})$ \\
\hline $1 \mathrm{mo}$ & $115.6 \pm 9.3$ & $51.6 \pm 46.8$ \\
$2 \mathrm{mo}$ & $197.2 \pm 36.1$ & $57.5 \pm 37.4$ \\
$4 \mathrm{mo}$ & $179.1 \pm 98.0$ & $130.2 \pm 82.0$ \\
$6 \mathrm{mo}$ & $277.8 \pm 79.6$ & $115.7 \pm 85.4$ \\
$12 \mathrm{mo}$ & $267.8 \pm 88.7$ & $158.2 \pm 107.8$ \\
Native aorta & $238.8 \pm 48.1$ & $427.2 \pm 64.8^{*}$ \\
\hline
\end{tabular}

${ }^{*} P<.05$ versus all other groups. 
shortcomings, including invasiveness, risk of contamination, and the need for preparation time, have led to hesitation in their clinical use. In this study, we used in situ cellularization as an alternative method that would be more suitable for clinical application because of its ready availability. ${ }^{14,15}$ As a candidate material for our TEVG, we focused our attention on a tissue-engineered patch with good cellular attachment properties that could provide a feasible microenvironment for vascular self-renewal. ${ }^{15}$ Cell counting proved that PGA/PLLA grafts had a much higher cellular affinity than ePTFE grafts designed for the same purpose. $^{18}$

The PGA/PLLA grafts demonstrated good in situ cellularization. The in vivo study produced the interesting observation that all the implanted PGA/PLLA grafts showed complete endothelialization, whereas this was never seen with any ePTFE graft, irrespective of the implantation period. After experiments on vascular wall reconstruction with a tissue-engineered patch, we previously reported that endothelialization was complete by 1 month after grafting. ${ }^{15}$ Similarly, in this study complete endothelialization was present by 1 month at least. Scanning electron micrography showed the endothelial integrity of the luminal surfaces, and immunohistochemical examination showed that the newly organized endothelium was accompanied by a basal membrane. It is well known that endothelialization yields antithrombogenic properties. ${ }^{9,10}$ The grafts were free of thrombus formation during the observation period despite the lack of antiplatelet and anticoagulant drugs. In ePTFE grafts, the direct exposure of ePTFE fibrils to the graft lumen and the irregularity of the graft surface at defective sites might cause local turbulence of intraluminal blood flow, resulting in an incidental

A
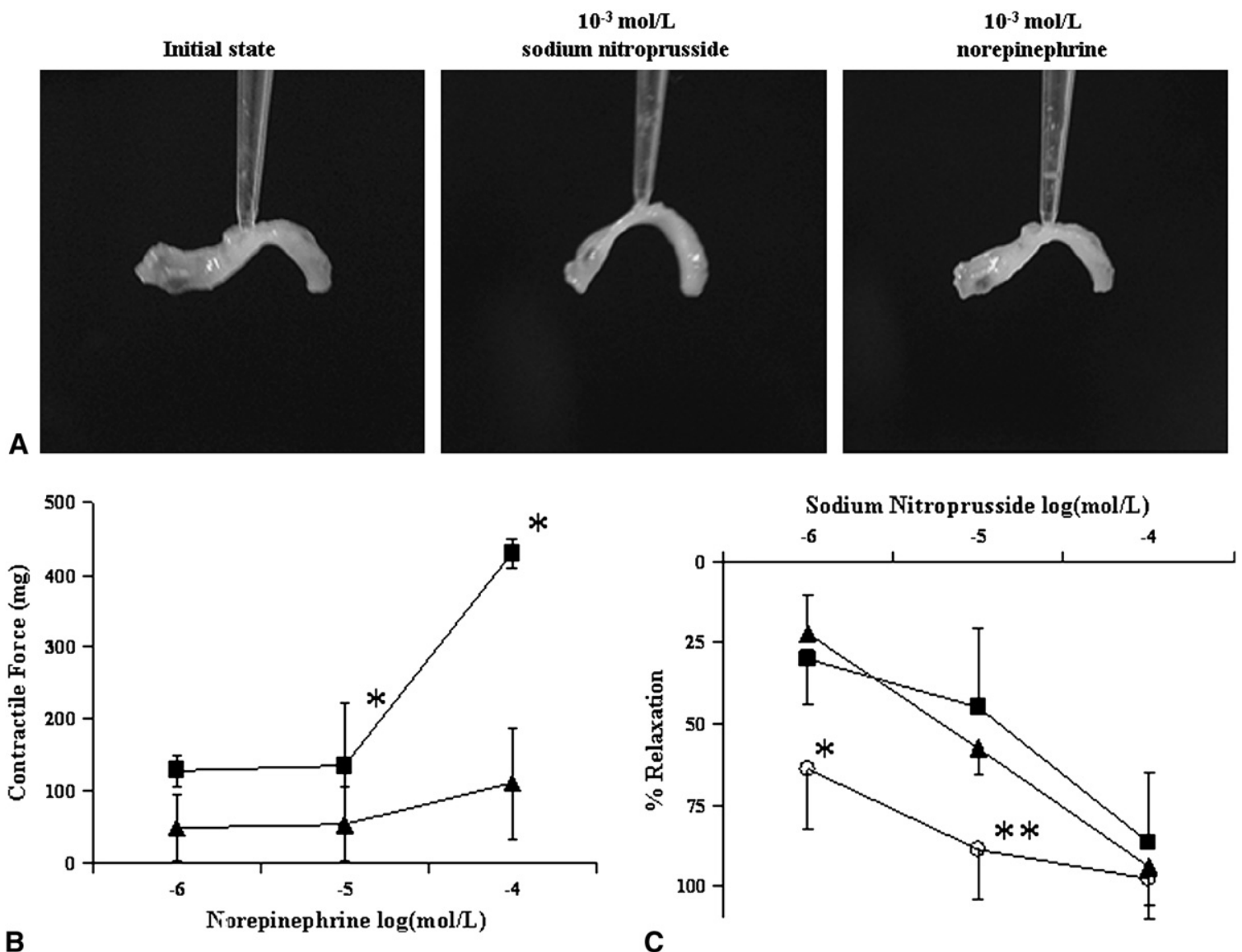

Figure 5. A, Qualitative study of vasomotor responsiveness with neotissue obtained from 2-month model. Suspended arch-shaped sample exhibited series of formation changes suggestive of relaxation and contraction on administration of sodium nitroprusside and norepinephrine, respectively. B, Contractile activities of 2-month (triangles) and 12-month (squares) models in isometric studies. Both models exhibited dose-response curves to norepinephrine. Asterisk indicates $\boldsymbol{P}<.05$ versus 2-month model. C, Relaxation responses of 2-month (triangles) and 12-month (squares) models and native aortas (circles) in isometric studies. Data represent percentage reduction in precontraction with $10^{-5}-\mathrm{mol} / \mathrm{L}$ norepinephrine. In each model, dose-dependent relaxation to sodium nitroprusside was demonstrated. Asterisk indicates $\boldsymbol{P}<.05$ versus 2 - or 12 -month model. Double asterisk indicates $\boldsymbol{P}<.05$ versus 12-month model. 
thrombotic event because of thrombogenicity or in an infection if a pathogen was present in host blood.

A population of numerous SMCs with parallel accumulation in the subendothelial layers, as seen in native media, was demonstrated. To determine whether the retrieved grafts had a physiologic vascular function, we tested the contractile and relaxation responses of the SMCs. At 2 months, the regenerated tissue already showed a reproducible pharmacologic vasomotor reaction. The contractility in response to norepinephrine increased in proportion to both the concentration of the agent and the implantation period. The magnitude of the maximal contractile force in the 12-month model was equal to or greater than that in other pretreated TEVGs reported on previously. ${ }^{7,8}$ In the relaxation studies with SNP, similar dose-response curves were found in all examined groups. The duration of implantation did not affect the relaxation of samples retrieved from PGA/PLLA grafts. These responses suggest that SMCs in the neomedia could acquire endothelium-independent vascular function to some extent as a result of being grown in an in situ bioreactor. In addition, we verified the existence of vasa vasorum in the neomedia, reflecting the neovascularization essential for tissue growth.

Biochemical examination showed that collagen in the neotissue was fully regenerated in the process of in situ cellularization at 6 months after grafting. Sufficient collagen to act as a framework might give the newly organized tissue dynamic strength against the systemic pressure. Histologic and biochemical evaluation, however, showed that the extent of elastin remodeling had not reached that of the controls during the experimental period. This implies that elasticity of the neotissue might be insufficient relative to native tissue. We speculate, on the basis of the morphologic observations, that tissue regeneration by host cells on PGA/PLLA grafts involving absorption of the biodegradable scaffolds was active in the early phase and that the basic vascular architecture was complete at 2 months after grafting. In the later phase, the regeneration process seemed gradually to become inactive, whereas neotissue remodeling, particularly by the extracellular matrix, progressed, as shown by the biochemical quantifications. As a result, the walls of our TEVGs were of almost constant thickness throughout the survey.

Considering the use of PGA/PLLA grafts for arterial intervention, we needed to evaluate their postimplantation durability through long-term follow-up. The strength of the PLLA layers, which are the major supporting factors in the prostheses, should be gradually reduced after grafting as a result of hydrolysis and perhaps biologic processes, including phagocytosis. In the in vivo implantation study, no case of rupture or aneurysm formation was observed. The maximal tensile strength of the remaining PLLA alone was even greater than that of the native tissue throughout our observations. Also, the firm connective tissue surrounding the grafts, which resulted from the wound healing process, would assist in reinforcing them. Consequently, it is thought that the actual strength of the implanted grafts would exceed that of native aortas. It remains unclear, however, whether the mechanical strength of a graft entirely replaced by autologous tissue can be resistant to systemic pressure, and therefore further long-term follow-up is required.

We selected the pig as an experimental model in this study because its hemodynamics resemble those of human beings. The systemic blood pressure of the animals was 100 to 140 $\mathrm{mm} \mathrm{Hg}$ in the systolic phase and 50 to $90 \mathrm{~mm} \mathrm{Hg}$ in the diastolic phase. The graft length of $30 \mathrm{~mm}$ was determined to avoid complete tissue regeneration simply by transanastomotic ingrowth of host cells. ${ }^{19}$ The detailed process of in situ cellularization has not been elucidated in this study. It is certain, however, that the scaffold provided by the newly developed graft would be a more suitable microenvironment for self-renewal of the vascular architecture than would a conventional prosthesis if tissue regeneration, including endothelialization, could not be achieved because of the predetermined graft length. We speculated on other mechanisms of in situ cellularization in which circulating progenitor cells would fall into the scaffold more easily, or transmural ingrowth of certain pericytes that guide vascular regeneration into the scaffold would occur more readily, with subsequent differentiation and proliferation according to the microenvironment. ${ }^{8,9}$ In this experimental model, the implanted grafts were grown in the native circulation system, which acted as an in situ bioreactor. We therefore speculate that the proper lining up of cells with high affinity on our TEVGs might be achieved by a mechanism analogous to the healing process in native vessels.

The limitations of our study are the lack of regenerated elastin and the residual PLLA layers. We speculate that the poor elastogenesis seen in implanted PGA/PLLA grafts might occur because the remaining outer, stiff PLLA layer prevents arterial pulsation and transmission of shear stress to the graft wall. To resolve these limitations, it would be necessary to modify the graft design so that the PLLA component is completely degraded in a relatively early phase after grafting or to apply substances that are known to accelerate elastin synthesis, such as retinoic acid or calcitriol. ${ }^{20,21}$

Considering the established use of artificial grafts for aortic surgery and the need for small-diameter prostheses, the complete and early endothelialization demonstrated by our TEVG is meaningful, particularly in the setting of smalldiameter vascular structures, such as peripheral arteries. In future, two ways to optimize the self-renewing mechanism should be considered. One is use of a TEVG that is completely replaced by autologous tissue. Assuming that the synthetic materials were fully degraded and that satisfactory remodeling of elastin was achieved, such a TEVG may have considerable compliance. This is of great importance, because the compliance of a graft, particularly of small diameter, is closely associated with its patency. ${ }^{22}$ Another possibility is a TEVG that is partially replaced by autologous 
tissue. To avoid concerns about time-dependent deterioration in the mechanical properties of PGA/PLLA grafts, nondegradable materials such as polyester, which can guarantee constant adequate strength, could be used as alternatives to PLLA. This kind of vascular graft would be expected to exhibit especially well-regulated endothelialization matched to the native vessel, reducing the risk of graft infection and providing durability adequate to withstand the arterial pressure throughout the host's life. This application is also likely to be suitable for medium- and large-diameter grafts, in which elasticity is not so important clinically.

In conclusion, a PGA/PLLA graft showed evidence of good in situ cellularization, including relatively early endothelialization and population with functioning SMCs, after implantation into porcine aorta. The graft was durable enough to withstand high-pressure conditions for at least 12 postoperative months, despite the presence of a residual PLLA layer, and also demonstrated the potential to acquire intrinsic physiologic vascular function. Our novel vascular graft would be a useful prosthesis even for arterial reconstruction, particularly in peripheral arterial surgery, and might provide a wide variety of therapeutic options in cardiovascular surgery.

We thank Mr Shigeru Matsumi for excellent technical assistance.

\section{References}

1. Edelman ER. Vascular tissue engineering: designer arteries. Circ Res. 1999;85:1115-7.

2. Christine ES, Jennie MB. Acellular vascular tissues: natural biomaterials for tissue repair and tissue engineering. Biomaterials. 2000;21:2215-31.

3. Langer R, Vacanti JP. Tissue engineering. Science. 1993;260:920-6.

4. Shin'oka T, Imai Y, Ikada Y. Transplantation of a tissue-engineered pulmonary artery. $N$ Engl J Med. 2001;344:532-3.

5. Shin'oka T, Matsumura G, Hibino N, Naito Y, Watanabe M, Konuma T, et al. Midterm clinical result of tissue-engineered vascular autografts seeded with autologous bone marrow cells. J Thorac Cardiovasc Surg. 2005;129:1330-8.

6. Sharp MA, Phillips D, Roberts I, Hands L. A cautionary case: the SynerGraft vascular prosthesis. Eur J Endovasc Surg. 2004;27:42-4.

7. Niklason LE, Gao J, Abbott WM, Hirschi KK, Houser S, Marini R, et al. Functional arteries grown in vitro. Science. 1999;284:489-93.
8. Campbell JH, Efendy JL, Campbell GR. Novel vascular graft grown within recipient's own peritoneal cavity. Circ Res. 1999;85:1173-8.

9. Kaushal S, Amiel GE, Guleserian KJ, Shapira OM, Perry T, Sutherland FW, et al. Functional small-diameter neovessels created using endothelial progenitor cells expanded ex vivo. Nat Med. 2001;7: 1035-40.

10. He M, Shirota T, Yasui H, Matsuda T. Canine endothelial progenitor cell-lined hybrid vascular graft with nonthrombogenic potential. J Thorac Cardiovasc Surg. 2003;126:455-64.

11. Hoerstrup SP, Cummings I, Lachat M, Schoen FJ, Jenni R, Leschka S, et al. Functional growth in tissue-engineered living, vascular grafts: follow-up at 100 weeks in a large animal model. Circulation. 2006; 114(1 Suppl):I159-66.

12. Opits F, Schenke-Layland K, Cohnert TU, Starcher B, Halbhuber KJ, Martin DP, et al. Tissue engineering of aortic tissue: dire consequence of suboptimal elastic fiber synthesis in vivo. Cardiovasc Res. 2004;63: 719-30.

13. Chen G, Sato T, Ushida T, Hirochika R, Tateishi T. Redifferentiation of dedifferentiated bovine chondrocytes when cultured in vitro in a PLGAcollagen hybrid mesh. FEBS Lett. 2003;542:95-9.

14. Iwai S, Sawa Y, Ichikawa H, Taketani S, Uchimura E, Chen G, et al. Biodegradable polymer with collagen microsponge serves as a new bioengineered cardiovascular prosthesis. J Thorac Cardiovasc Surg. 2004; 128:472-9.

15. Iwai S, Sawa Y, Taketani S, Torikai K, Hirakawa K, Matsuda H. Novel tissue-engineered biodegradable material for reconstruction of vascular wall. Ann Thorac Surg. 2005;80:1821-7.

16. Angus JA, Wright CE. Techniques to study the pharmacodynamics of isolated large and small blood vessels. J Pharmacol Toxicol Methods. 2000;44:395-407.

17. Matsumoto T, Tsuchida M, Sato M. Change in intramural strain distribution in rat aorta due to smooth muscle contraction and relaxation. Am J Physiol. 1996;271(4 Pt 2):H1711-6.

18. Clowes AW, Kirkman TR, Reidy MA. Mechanisms of arterial graft healing. Rapid transmural capillary ingrowth provides a source of intimal endothelium and smooth muscle in porous PTFE prostheses. Am J Pathol. 1986;123:220-30.

19. Greisler HP, Dennis JW, Endean ED, Ellinger J, Buttle KF, Kim DU. Derivation of neointima in vascular grafts. Circulation. 1988;78(3 Pt 2):I6-12.

20. Tukaj C, Kubasik-Juraniec J, Kraszpulski M. Morphological changes of aortal smooth muscle cells exposed to calcitriol in culture. Med Sci Monit. 2000;5:668-74.

21. Hayashi A, Suzuki T, Tajima S. Modulations of elastin expression and cell proliferation by retinoids in cultured vascular smooth muscle cells. J Biochem. 1995;117:132-6.

22. Haruguchi $\mathrm{H}$, Teraoka S. Intimal hyperplasia and hemodynamic factors in arterial bypass and arteriovenous grafts: a review. J Artif Organs. 2003;6:227-35 


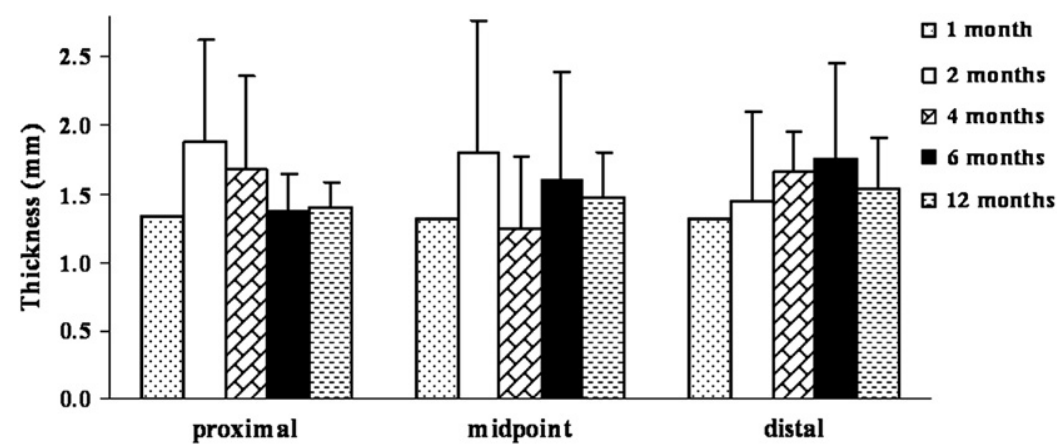

Figure E1. Wall thickness of neotissue formed in implanted PGA/PLLA grafts. Thickness was measured at three points: $5 \mathrm{~mm}$ from proximal and distal anastomoses, respectively, and at midpoint of graft. In all groups, mean value at each measured point was approximately $1.5 \mathrm{~mm}$.

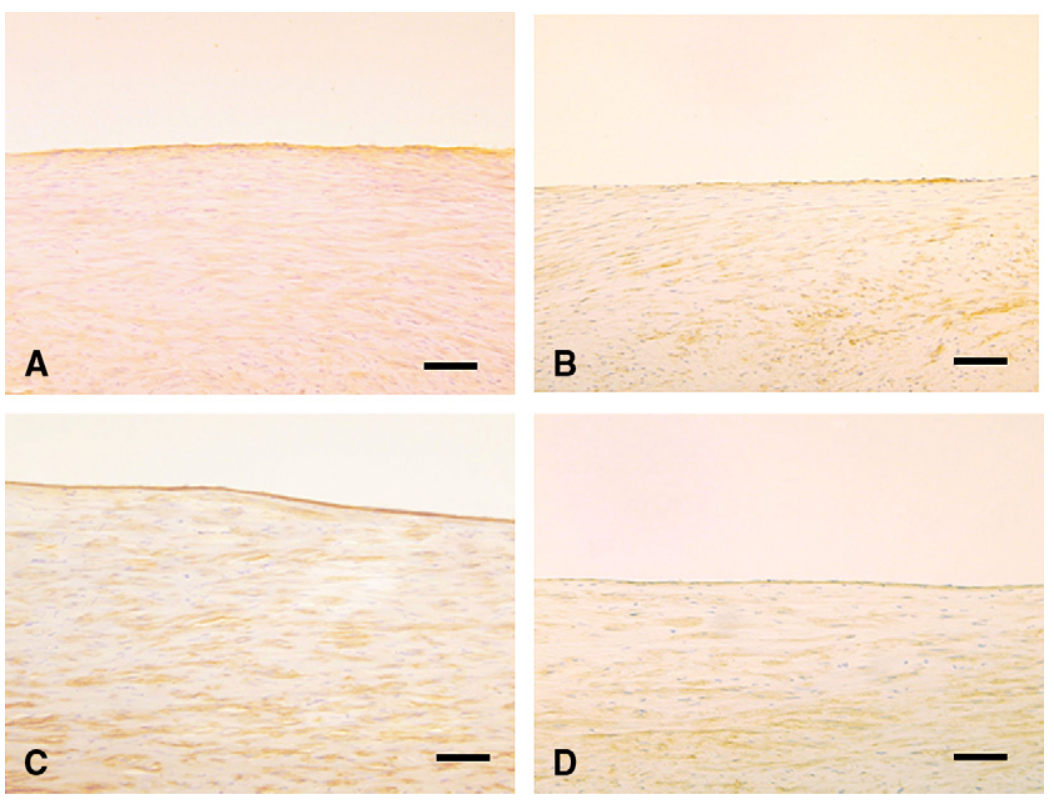

Figure E2. Immunohistochemical examination for collagen type IV 1 (A), 2 (B), 4 (C), and 6 (D) months after implantation. Positively stained thin layers just below endothelium and scattered positive staining in subendothelial layers were seen. Original magnification $\times 100$; scale bar, $100 \mu \mathrm{m}$.

TABLE E1. Time course of regeneration process in polyglycolic acid/poly-L-lactic acid grafts

\begin{tabular}{|c|c|c|c|c|c|}
\hline & $1 \mathrm{mo}$ & $2 \mathrm{mo}$ & $4 \mathrm{mo}$ & $6 \mathrm{mo}$ & $12 \mathrm{mo}$ \\
\hline Endothelialization & + & + & + & ++ & ++ \\
\hline Smooth muscle cells & + & + (functioning) & + & + & ++ \\
\hline Vasa vasorum & + & + & + & + & + \\
\hline Polyglycolic acid & + & \pm & - & - & - \\
\hline Poly-L-lactic acid & + & + & $\downarrow$ & $\downarrow \downarrow$ & $\downarrow \downarrow$ \\
\hline
\end{tabular}

